\title{
New Delay-based Fast Retransmission Policy for CMT-SCTP
}

\author{
Lal Pratap Verma \\ Department of Computer Science and Engineering, \\ Jaypee University of Engineering and Technology, Guna, Madhya Pradesh, (INDIA) \\ E-mail: er.lpverma1986@gmail.com \\ Varun Kumar Sharma, Mahesh Kumar \\ Department of Computer Science and Engineering, \\ Jaypee University of Engineering and Technology, Guna, Madhya Pradesh, (INDIA) \\ E-mail: \{varunksharma.102119.cse, mahesh.chahar\}@gmail.com
}

Received: 20 April 2017; Accepted: 06 July 2017; Published: 08 March 2018

\begin{abstract}
Concurrent Multipath Transfer (CMT) uses multi-homing feature of Stream Control Transmission Protocol (SCTP) to transfer data concurrently over the multiple paths. CMT provides bandwidth aggregation, fault tolerance, and reliability in multipath data transfer. In multipath data transmission, each path has different delay and bandwidth. Therefore, destination receives unordered data which causes receiver buffer blocking and unwanted congestion window ( $c w n d$ ) reduction. Both the problem degrades the CMT performance significantly. Thus, this paper proposes a new delay-based fast retransmission policy to adjust the transmission rate of each path according to path delay. Simulation results show that the proposed approach achieves better throughput, reduces the number of the timeout and improves the cwnd growth. The proposed approach improved throughput up to $16 \%$ in variable packet loss and $18 \%$ in variable network delay environment.
\end{abstract}

Index Terms-SCTP, CMT, Multipath, Multi-homing, Congestion window.

\section{INTRODUCTION}

A device is having more than one network interface, like Laptop or smartphones, is called multi-homed device. The multi-homing offers a pair of devices to establish the logical connection over the multiple interfaces [1]. The advantage of multi-homing devices is that they provide the backup path in case of network failure. The SCTP [1] is a transport layer protocol, provide message-oriented, full duplex, connection-oriented, and multi-homing services. SCTP offers elective reliability and ordering in a stream, multi-homing, multi-streaming and protection against SYN attacks. SCTP assumes one IP as a primary path while remaining treated as the secondary path. SCTP uses the transmission sequence number (TSN) to ensure the ordered data delivery. To take advantage of multihoming, Iyengar et al. [2] proposed a CMT to transfer the data over the multiple paths concurrently. It provides bandwidth aggregation, robustness, and reliability in multipath data transfer. However, due to dissimilar path delay and bandwidth, multipath data transmission leads unordered data packet delivery at the receivers end. It causes unnecessary retransmissions, unwanted cwnd reduction, and receiver buffer blocking [3]. To minimize the receiver buffer blocking, Iyengar et al. [2] suggested the five retransmission path selection policies. However, these policies do not improve the buffer blocking in dissimilar bandwidth and delay network. To mitigate the unordered data chunk delivery, we suggested a new delay-based adaptive data chunk scheduling policy [24] to distribute data over the multipath according to the path delay and available bandwidth. The suggested policy improves the network utilization but still suffers from unwanted fast retransmission problem.

During fast retransmission [2], whenever CMT receives four duplicate SACKs, treats it as network congestion, reduces the cwnd and ssthresh to half of the current $c w n d$. However, destination also sent the SACKs when it receives unordered data. Therefore, the cause of unordered data delivery is dissimilar delay and bandwidth of each path. The maximum numbers of duplicate SACKs are generated due to unordered data delivery. Thus, the blind half reduction in cwnd is not appropriate for CMT and CMT-PF because it degrade the performance of CMT and CMT-PF significantly [3-4].

Therefore, this paper proposes a new delay-based fast retransmission policy to mitigate the receiver buffer blocking and cwnd growth problem. The new approach uses SRTT (Smooth Round Trip Time) as congestion window reduction factor. This factor reduces the $c w n d$ in the small amount when RTT (round trip time) is small and reduces the $c w n d$ in the significant amount when RTT is large.

The rest of the paper is organized as follows: Section 2 presents literature reviews of various CMT policies while section 3 presents a new delay-based fast retransmission technique for CMT. The performance evaluation of the proposed approach is presented in section 4 while section 
5 concludes the overall performance of proposed method.

\section{RELATED WORK}

Iyengar et al. [2] identified the spurious retransmission problem of CMT and proposed a solution called Split Fast Retransmit (SFR) algorithm. It improves the performance of CMT concerning retransmission but suffers from unnecessary $c$ wnd reduction when destination receives unordered data chunk due to dissimilar path delay and bandwidth. Iyengar et al. [2] suggested another algorithm, which maintains the separate congestion window for each destination to grow independently. It improves the $c w n d$ growth but has the same problem of unnecessary cwnd reduction. The SCTP decrease the acknowledgment traffic by delaying acknowledgment until at least two can be sent collectively [2]. However, SCTP sends an immediate acknowledgment, when it receives unordered data chunk. Because of frequent unordered data chunk delivery, the reordering acknowledgment increase regularly. Delayed Ack for CMT (DAC) was included into SFR to minimize the acknowledgment traffic [2].

Ye et al. [4] proposed IPCC-SCTP to reduce the false retransmissions. It uses the unique path sequence number (PSN) for each path, which decides the ordered or unordered delivery of chunk for each destination. IPCCSCTP improves the retransmission but suffers from buffer blocking problem. Dreibholz et al. [5] suggested a Sender Buffer Splitting approach which splits the sender buffer according to the number of paths. The author claims that proposed approach improved receiver buffer blocking but suffers from local blocking due to the dissimilarity of the path. Authors $[3,6]$ investigated the CMT and identified unnecessary fast retransmissions, crippled window growth, excessive network traffic; receive buffer blocking and naive scheduling problems.

Natarajan et al. [7] identified receiver buffer blocking problem due to path failure and suggested the solution (a new state for each destination) called PotentiallyFailed (PF). This state indicates that the destination is not reachable due to congestion or link failure. Thus, all the new data transmitted over the available alternate path. It minimizes the packet loss due to link failure but suffers from receiver buffer blocking due to dissimilar bandwidth and delay of each path.

The CMT suffers from receiver buffer blocking due to dissimilar path delay and bandwidth causes unordered data chunk delivery. Yilmaz et al. [8] suggested a nonrenegable selective acknowledgment (NR-SACKs) to free the receiver buffer. The NR-SACK simply removes the segment without bothering about reordering. Shailendra et al. [9] suggested an MPSCTP (Multipath SCTP) as a solution to unnecessary retransmission and window growth. The author claims better throughput and reduced retransmissions but suffers from buffer blocking problem. Shailendra et al. [10] proposed delay-based transmission adjustment policy to reduce the average packet delay of over the multiple paths. It minimized the buffer blocking problem but suffers from low bandwidth utilization. Shailendra et al. [11] suggested a Tx-CWND retransmission destination selection policy to improve the performance of MPSCTP in terms of receiver buffer blocking. Xu et al. [12] suggested a Quality-aware adaptive concurrent multipath data transfer in heterogeneous wireless networks (CMT-QA) to send data according to path quality. However, path quality estimation provides incorrect path quality value due to dissimilar path delay and bandwidth always have variable trends.

Authors also investigated soft computing based approaches [20-22, 29-30] to optimize network performance in wireless network. However, Thang and Tao [31] investigated the IPv6 routing protocol performance for Wireless Sensor Networks (WSN). Sharma and Kumar [23] suggested an adaptive congestion control scheme in mobile ad-hoc networks to improve the utilization of network.

MP-TCP [13] is another key connection-oriented protocol supports multi-homing. Likewise, SCTP does. MP-TCP works on the principal of distributing traffic over multiple paths. MP-TCP provides transparency in between top layer (application) to multiple connections. Moreover, MP-TCP works perfectly fine with the integrations of middle-boxes in today's Internet architecture [14-18]. MP-TCP offers better performance (comparing with conventional TCP) with data segments tearing middle-boxes in Internet's architecture. Consequently, MP-TCP offers better deployment capability with modern Internet architecture. In recent years, many of the un-coupled (independent congestion control between different sub-flows) strategies [25-26] were introduced. Nevertheless, the policy of controlling congestion independently (by sub-flows) leads to unfairness issue in the system. For this, MP-TCP introduces adaptive coupled congestion control policy by appropriately transforming congestion window growth policy concerning each sub- flow's network state [2728].

Recently, various techniques have been presented to improve the MPTCP performance [14-18]. However, during the fast retransmission, all the suggested techniques reduce the $c w n d$ to half of the current cwnd blindly which significantly degrade the performance of MPTCP.

\section{PROPOSED WORK}

In this section, we present a new delay-based fast retransmission policy to minimize the $c$ wnd growth problem in multipath concurrent data transfer using CMT-SCTP. The multipath concurrent data transfer sends the data over the multiple paths while each path has different bandwidth and delay. Due to dissimilar path characteristics, data packet arrived out-of-order at the destination. When destination receives unordered data packet, it immediately sends gap information to the source. Four-time continuous reception of gap report concludes congestion on the path. Thus, source reduces the $c w n d$ and ssthresh to half of current cwnd. However, 
network is not congested. Therefore, such reduction causes significant performance degradation while path is not congested.

When network congestion increases, RTT also increases, whereas unordered data chunk delivery may not increase the RTT. If, we include path delay as a factor of $c w n d$ reduction, then it will control the reduction in cwnd and ssthresh appropriately instead of reducing to half blindly.

\section{A. Effect of delay on cwnd reduction}

The path delay plays a significant role in multipath data transfer because each path has different bandwidth and delay. Each path delay varies when path traffic intensity changes. If we reduce the $c w n d$ according to path delay variation, then it may minimize the $c w n d$ growth problem. The delay of the path is large if path is having high traffic intensity while delay is small when path has normal traffic intensity. If, reduction of the $c$ wnd is made using the product of current path delay and $c w n d$, then it reduces the cwnd in a small amount when delay is small and reduces $c w n d$ with large amount when delay is large.

Let, RTT and cwnd is the delay and congestion window of the path-1. According to proposed approach, cwnd can be reduced by the product of path delay and cwnd. Therefore, the formula of cwnd reduction when path-1 has normal traffic intensity is as:

$$
c w n d_{i+1}=c w n d_{i}-\left(c w n d_{i} \times R T T_{i}\right)
$$

Let, RTT of path-1 is $\mathrm{RTT}_{1}$ when path is not congested then Eq. (1) is as

$$
\text { cwnd }_{i+1}=\text { cwnd }_{i}-\left(\text { cwnd }_{i} \times R T T_{1}\right)
$$

As congestion increases the RTT of the path also increases. Therefore, let the RTT of path-1 is $\mathrm{RTT}_{2}$ when network is having high traffic intensity. Thus, the $\mathrm{RTT}_{2}$ must be greater than $\mathrm{RTT}_{1}$. Therefore, cwnd reduction to be done according to Eq. (3) is as:

$$
c w n d_{i+1}=c w n d_{i}-\left(c w n d_{i} \times R T T_{2}\right)
$$

If, $\mathrm{RTT}_{2}>\mathrm{RTT}_{1}$. Then, the product of $c w n d_{i}$ and $\mathrm{RTT}_{2}$ is also greater than the product of $c w n d_{i}$ and $\mathrm{RTT}_{1}$. Hence, the reduction amount in cwnd is as

$$
\mathrm{cwnd}_{i} \times R T T_{2}>\mathrm{cwnd}_{i} \times R T T_{1}
$$

This relation shows that when delay is large, reduction in cwnd is large while reduction is small when delay is small.

\section{B. Path delay estimation}

CMT uses round trip time (RTT) to estimate the delay of the each path. The estimation of RTT of each path includes queuing delay, transmission delay, processing delay, and propagation delay as:

$$
\begin{gathered}
R T T_{\min }=P_{d}+P_{s}+T_{d}+Q_{\min } \\
R T T_{i}=P_{d}+P_{s}+T_{d}+Q_{i}
\end{gathered}
$$

where, $\mathrm{RTT}_{\mathrm{i}}$ is a current RTT, $\mathrm{RTT}_{\min }$ is a minimum RTT, $\mathrm{P}_{\mathrm{d}}$ is propagation delay, $\mathrm{Td}$ is a transmission delay, $\mathrm{Ps}$ is the processing delay, $\mathrm{Q}_{\min }$ is a minimum queuing delay, and $\mathrm{Q}_{\mathrm{d}}$ is a current queuing delay of path. Our proposed method uses average path delay to reduce the error in RTT estimation. For average delay estimation, we use SRTT (smooth round trip time) can be estimated as:

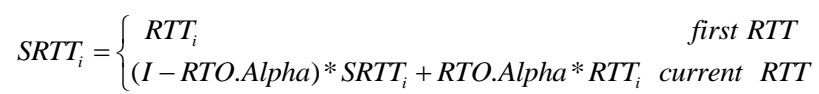

where, the recommended value of RTO.Alpha is 0.25 [1], $\mathrm{RTT}_{\text {min }}$ is the first RTT and $\mathrm{RTT}_{\mathrm{i}}$ is the current RTT measured by source.

\section{Congestion window reduction policy}

Let $\mathrm{P}_{\mathrm{i}}=\left\{\mathrm{P}_{1}, \mathrm{P}_{2}, \mathrm{P}_{3} \ldots \ldots \ldots \mathrm{P}_{\mathrm{n}}\right\}$ be the paths used for multipath transmissions, and the round trip delay of each path is defined as $D_{i}=\left\{D_{1}, D_{2}, D_{3} \ldots \ldots \ldots D_{n}\right\}$. If delay of $i^{\text {th }}$ path changes, it means that traffic on the path also changes. If we include path delay as a factor of $c w n d$ reduction, then it will control the reduction in $c w n d$ and ssthresh appropriately instead of reducing to half blindly. Thus, the proposed method includes the current path's cwnd and SRTT as a cwnd reduction factor. This factor has been independently estimated for each path while receiving four duplicate SACKs. It reduces the $c w n d$ of current path with large amount if congestion occurs while reduces with small amount in case of unordered data chunk delivery.

$$
\begin{gathered}
\text { ssthresh }=\max \left(\text { cwnd }_{i}-\left(\text { cwnd }_{i} \times S R T T_{i}\right), 4 \times M T U\right) \\
\text { cwnd } d_{i}=\text { ssthresh }
\end{gathered}
$$

where, MTU is the maximum transmission unit of SCTP. Eq. (7) and (8) show the formula for cwnd and ssthresh reduction. The algorithm of proposed fast retransmission policy is shown in Algorithm-1. The Algorithm-1 have two method, first is fast retransmission algorithm and second one is retransmission timeout (RTO) algorithm. Fast retransmission algorithm adjusts the transmission 
rate of path when source receives four duplicate SACKs. However, retransmission timeout algorithm adjusts the transmission rate of the path when retransmission timer expires. In multi-homing sinario, source calculates a separate RTO for each destination.

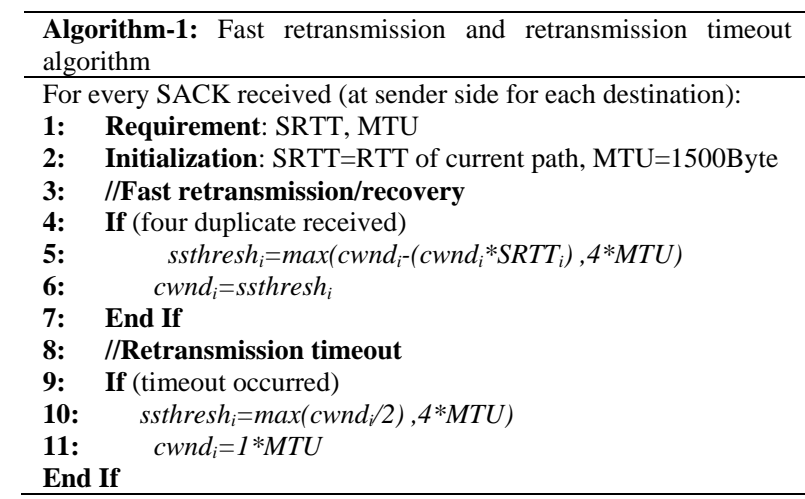

\section{Performance Evaluation}

In this section, we compare the performance of proposed retransmission policy with well known CMT [2] fast retransmission policy. The whole simulation has been performed by using NS-2.35 [19]. Fig. 1 shows the network topology used for simulation. The topology has one SCTP source with two network interfaces S1\&S2, and one SCTP destination with two network interfaces D1\&D2. Initial bandwidth and delay of each link are shown in Fig. 1. The delay and bandwidth of each link may change according to simulation requirements. In this simulation setup, path-1 has fixed packet loss rate $1 \%$, while path-2 has variable packet loss rate which varies from $1 \%$ to $10 \%$. The SCTP source connected with FTP traffic generator and simulation time of this setup is $200 \mathrm{sec}$

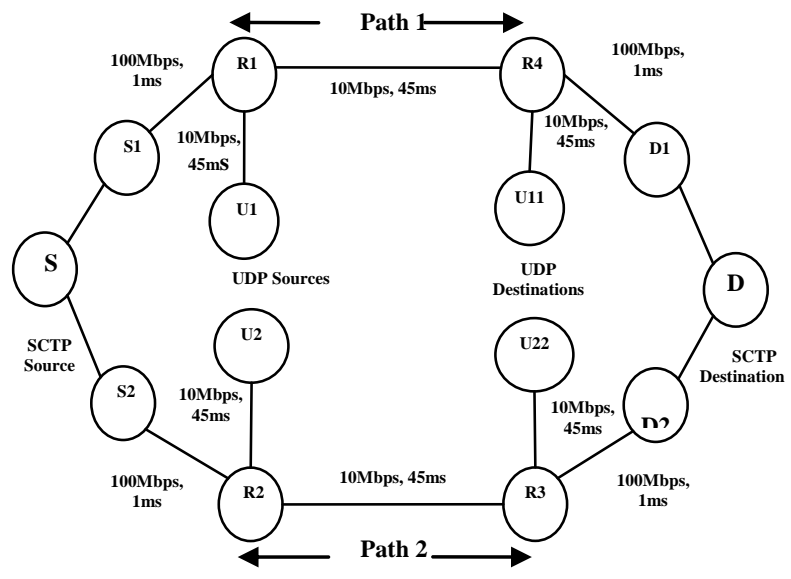

Fig.1. Simulation topology

The simulation topology also has two UDP sources U1, U2 and two UDP destinations U11, U22 respectively. The $\mathrm{U} 1$ and $\mathrm{U} 11$ are connected to router R1 and R4 while $\mathrm{U} 2, \mathrm{U} 22$ are connected to $\mathrm{R} 2$ and $\mathrm{R} 3$ respectively. This simulation setup is configured with drop tail queuing policy and default queue size is 50 packets. This simulation setup configured with recommended RTXCWND retransmission path selection policy.

Fig. 2, 3 and 4 show the analysis of throughput, average throughput and retransmission timeout of proposed method, CMT and CMT-PF. In this simulation, receiver buffer size is $64 \mathrm{~KB}$; simulation time is 200 seconds and packet loss rate of path-2 varies from $1 \%$ to $10 \%$. Rest of the network configuration is according to Fig. 1.

Fig. 2 demonstrates the throughput variation of CMT variants with variable packet loss rate. It shows that as packet loss rate increases the throughput of all CMT variants decreases. CMT and CMT-PF show the similar and linear throughput degradation because they use same cwnd and ssthresh reduction policy when congestion occurs, or unordered data chunk receives by destination. However, proposed method uses delay-based cwnd and ssthresh reduction policy which reduce the cwnd and ssthresh according to delay of the path. When path delay variation is large, it means that the traffic intensity is high, and if path delay variation is small, it means traffic is smooth. Thus, the proposed method use delay as factor of cwnd reduction which directly affect the cwnd reduction amount. Therefore, the proposed method shows the better cwnd growth and throughput for each packet loss rate.

Fig. 3 shows the average throughput of CMT variants with variable packet loss rate. It shows that CMT has least utilization as compared to CMT-PF and proposed method. However, the proposed method achieves improved throughput as compared to CMT and CMT-PF. The proposed method throughput improvement is $16 \%$ as compared to CMT and $15 \%$ as compared to CMT-PF.

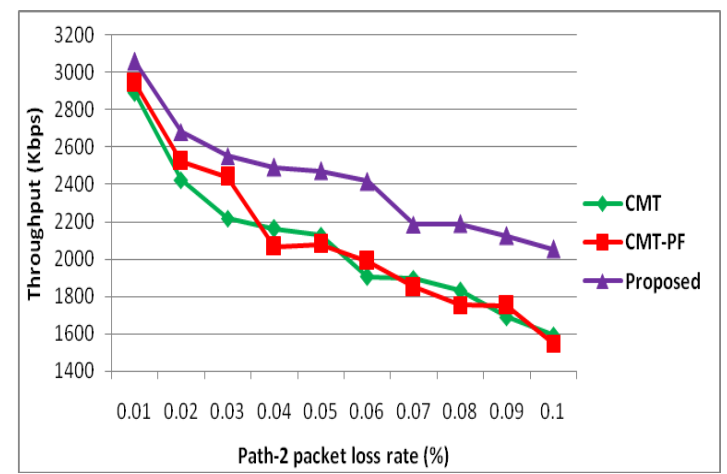

Fig.2. Packet loss rate Vs throughput

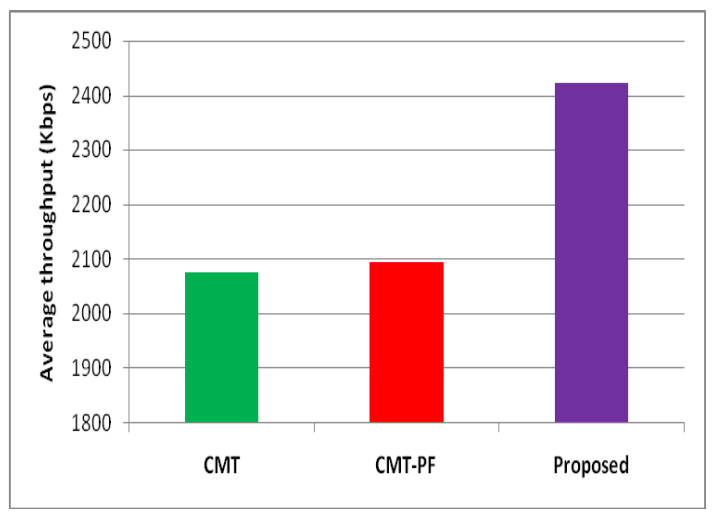

Fig.3. Average throughput of CMT variants 
We also estimated the confidence interval for this simulation result. For $95 \%$ confidence level, the confidence interval of proposed method, CMT and CMTPF are 2234.96-2611.63, 1837.27-2311.50 and 1830.402357.39 respectively. The confidence interval of all the CMT variants demonstrates that proposed method has better confidence interval as compared to CMT and CMT-PF.

Fig. 4 shows the average retransmission timeout of proposed method, CMT, and CMT-PF. It shows that CMT has highest number of timeout while proposed method shows the least number of timeout as compared to CMT and CMT-PF. It confirms that the delay-based cwnd and ssthersh reduction policy is a better approach as compared to halve the cwnd and ssthresh blindly. The proposed method average timeout improvement is $19 \%$ as compared to CMT and 6\% as compared to CMT-PF. We also calculate the confidence interval for this simulation results. For $95 \%$ confidence level, the confidence interval of proposed method, CMT and CMT-PF are 7.74-16.45, 10.84-19.35 and 8.27-17.72. It is evident from confidence interval that proposed method has lower confidence interval concerning timeout as compared to CMT and CMT-PF.

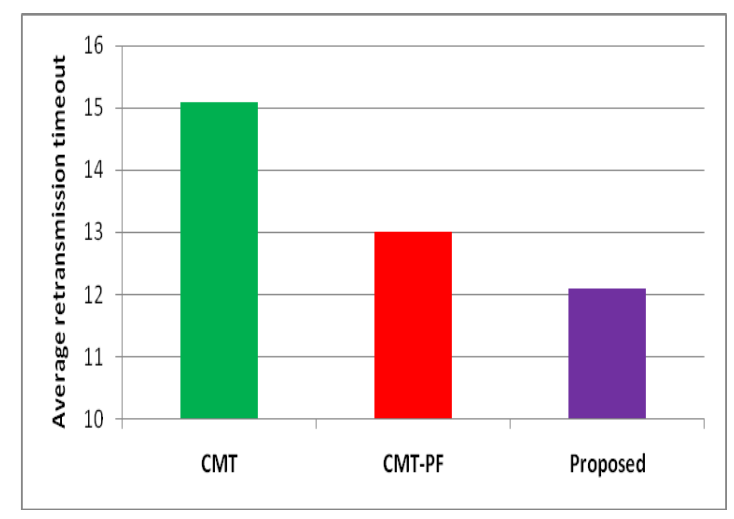

Fig.4. Average retransmission timeout of CMT variants

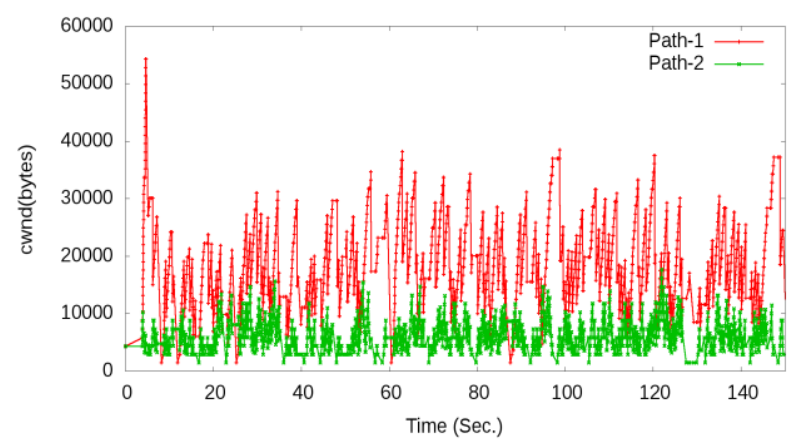

(a) CMT

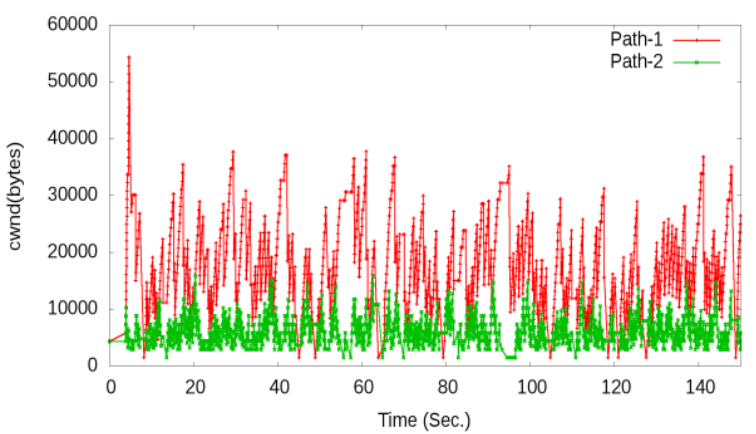

(b) CMT-PF

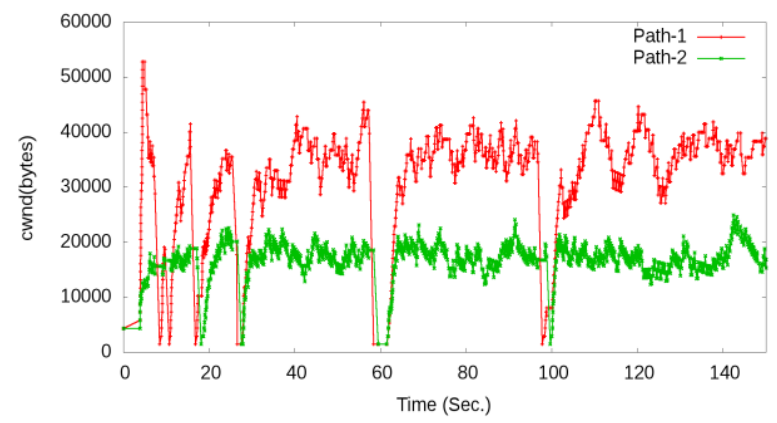

(c) Proposed

Fig.5. Congestion window growths Vs time while packet loss rate of path-1 is $1 \%$ and path-2 is $10 \%$ (a) CMT (b) CMT-PF (c) Proposed

Fig. 5 (a)-(c) show the cwnd growth of proposed method, CMT, and CMT-PF while path-1 has $1 \%$ and path-2 has $10 \%$ packet loss rate. In this simulation setup, simulation time is 150 seconds and rests of the configuration parameters are same as given in Fig.1. This simulation study demonstrates the cwnd growth and reduction when packet loss or timeout occurs. The CMT and CMT-PF reduce the cwnd and ssthresh to half of current $c$ wnd to adjust the transmission rate when source receives four duplicate SACKs. Therefore, CMT and CMT-PF suffers from cwnd growth problem. The proposed method uses the delay-based cwnd reduction approach to adjust the transmission rate. Therefore, proposed method reduces the $c w n d$ is the small amount when the reason of cwnd reduction is unordered data chunk delivery. However, proposed method reduces the $c$ wnd in the large amount when congestion occurs. Thus, the proposed method achieves better $c$ wnd growth as compared to CMT and CMT-PF.

In next simulation, we analyze the effect of variable path delay on throughput and retransmission timeout. In this simulation setup, RTT of path-1 (100ms) remains constant while path-2 has variable RTT varies from 50$400 \mathrm{~ms}$. The packet loss rate of path-1 is $1 \%$ while path-2 has $5 \%$ packet loss rate. Rest of the simulation configuration remains same according to Fig. 1. 


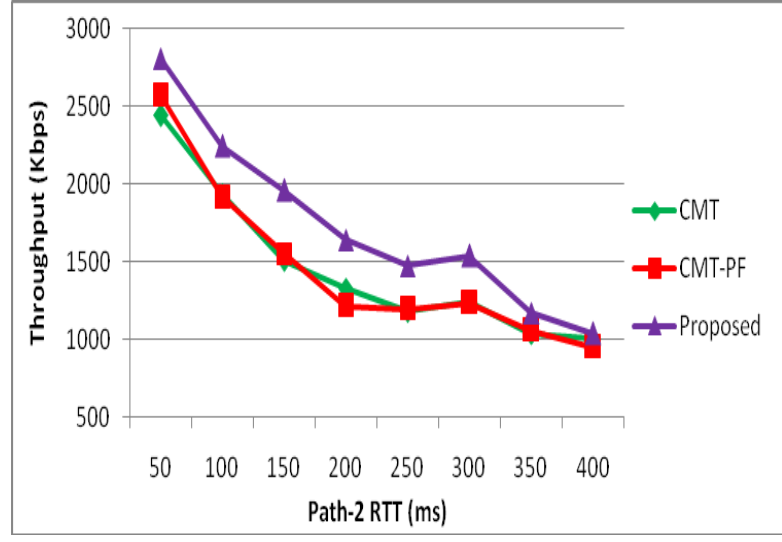

Fig.6. RTT Vs Throughput

Fig. 6 shows the throughput variation of CMT variants in variable RTT network environment. It demonstrates that as RTT increases, the throughput of the CMT variants decreases. The CMT and CMT-PF show the similar and linear trend in throughput drop. However, the proposed method demonstrates the higher throughput as compared to CMT and CMT-PF. The proposed method use path delay as $c w n d$ reduction factor which reduces the cwnd according to path traffic conditions. On the other hand, CMT and CMT-PF reduce the cwnd to half of current cwnd blindly. As a result, proposed method achieves better throughput as compared to CMT and CMT-PF. The proposed method average throughput improvement is $18.83 \%$ as compared to CMT and $18.64 \%$ as compared to CMT-PF. Fig.7 shows the average retransmission timeout of CMT variants in variable RTT network. It demonstrates that the CMT-PF suffers from more timeout as compared to CMT. However, the proposed method has less timeout as compared to CMT and CMT-PF due to its delay-based cwnd reduction policy. The proposed method average timeout improvements are $23 \%$ and $34 \%$ as compared to CMT and CMT-PF respectively.

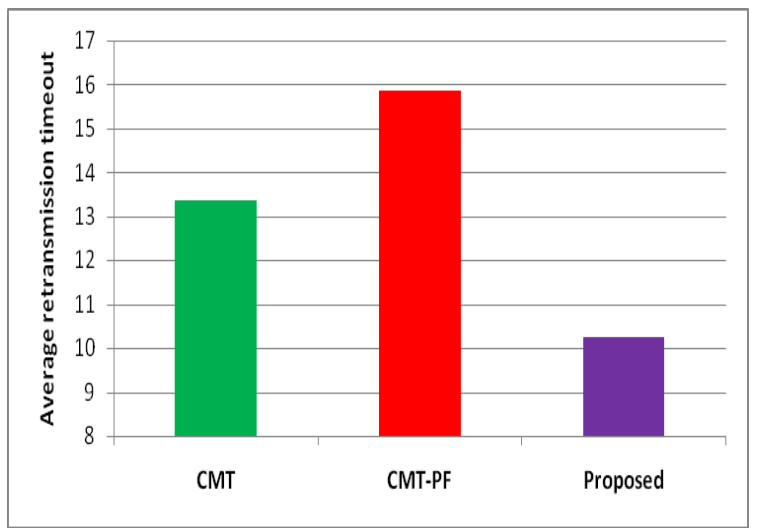

Fig.7. Average retransmission time of CMT variants

In another simulation, we analyze the effect of different receiver buffer (rbuf) on the performance of CMT variants. Fig.8(a)-(c) show the throughput of CMT, CMT-PF and proposed method with receiver buffer $32 \mathrm{~KB}, 64 \mathrm{~KB}$, and $128 \mathrm{~KB}$. In this simulation setup, the packet loss rate of path-1 and path-2 are $1 \%$ and $5 \%$.
However, the propagation delay of path-1 and path-2 are $50 \mathrm{~ms}$ and $150 \mathrm{~ms}$ respectively. Rest of the simulation configurations remains same according to Fig.1.

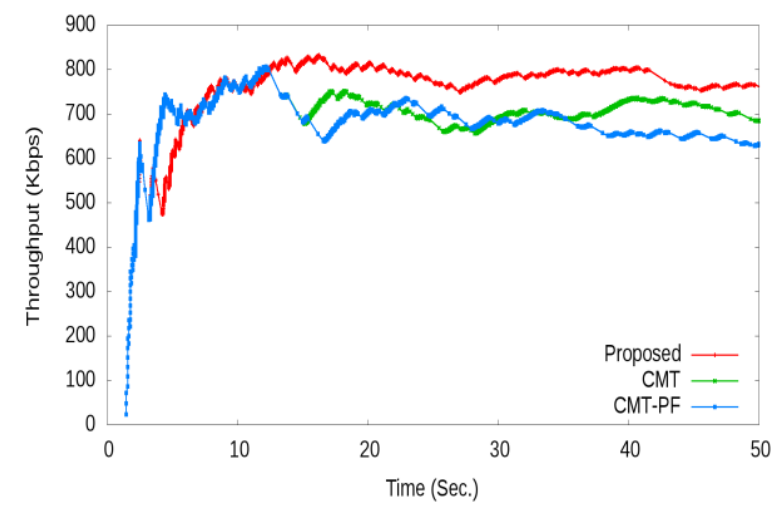

(a) $32 \mathrm{~KB}$ rbuf

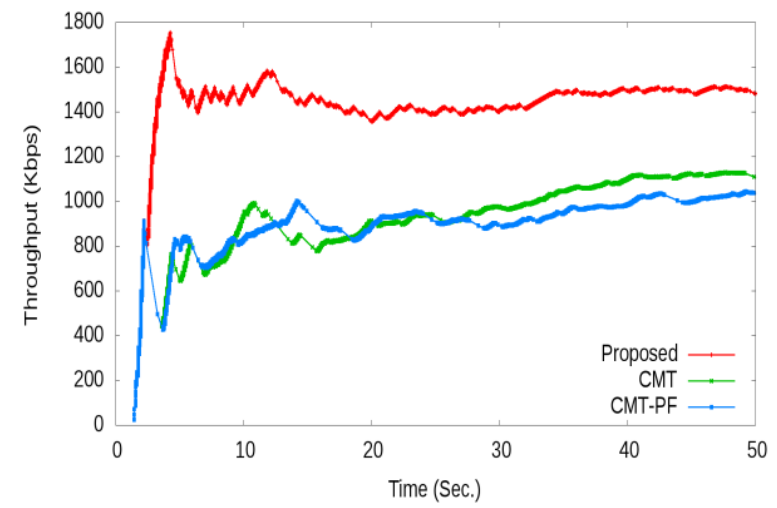

(b) $64 \mathrm{~KB}$ rbuf

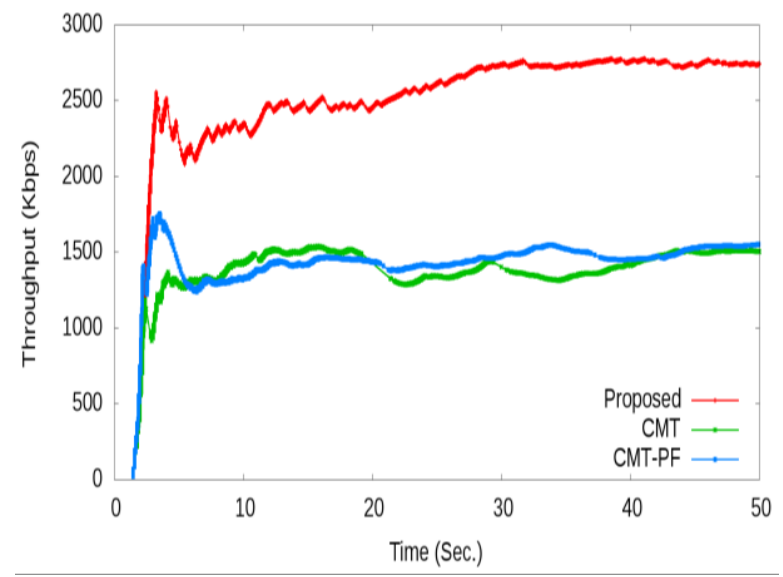

(c) $128 \mathrm{~KB}$ rbuf

Fig.8. Comparison of throughput using different receiver buffer sizes

It has been observed from Fig. 8(a)-(c), that the throughput of all CMT variants increases with the increase of receiver buffer size. At the start, the throughput of CMT variants increases rapidly because CMT variants probe the network capacity. After reaching network capacity, the throughput of CMT variants experiences variation due to packet loss detection (caused by congestion or unordered data delivery), then $c w n d$ adjustment and fast retransmission. The proposed method differentiates the cwnd adjustment cause by either packet loss or unordered data delivery using delay-based $c w n d$ 
adjustment policy. Therefore, the proposed method reduces the cwnd in a small amount when packet loss detected due to unordered data delivery while cwnd reduction is large when packet loss detected due to congestion. Such type of cwnd reduction improves the network utilization and reduces the timeout. As a result, the proposed method achieves better throughput as compared to CMT and CMT-PF for all receiver buffer size.

\section{CONCLUSION}

In this paper, we proposed a novel fast retransmission approach for CMT to adjust the cwnd and ssthresh based on path delay. The proposed approach uses the product of SRTT and cwnd as a cwnd reduction factor. This approach reduces the cwnd in the large amount when network is congested while it reduces the $c$ wnd in the small amount when reduction caused by unordered data chunk delivery. The simulation results show that the proposed method achieves better throughput, reduces the retransmission timeout and has better $c w n d$ growth as compared to CMT and CMT-PF. The proposed method average throughput improvement is $16 \%$ in variable packet loss rate and $18 \%$ in variable path delay environment.

\section{REFERENCES}

[1] R. Stewart, "Stream control transmission protocol", RFC4960, The Internet Engineering Task Force, 2007. https://tools.ietf.org/html/rfc4960.

[2] J. Iyengar, P. Amer and R. Stewart "Concurrent Multipath Transfer Using SCTP Multihoming Over Independent End-to-End Paths," IEEE/ACM Transactions on Networking, vol. 14, pp. 951-964, 2006.

[3] TD. Wallace and A. Shami "A Review of Multihoming Issues using Streaming Control Transmission Protocol," IEEE Communication Surveys \& Tutorials, vol. 14, pp 565-578, 2012.

[4] G. Ye, T. Saadawi and M. Lee "IPCC-SCTP: An enhancement to the standard SCTP to support multihoming efficiently," IEEE International Conference on Performance, Computing, and Communications, pp. 523530, 2004.

[5] T. Dreibholz, M. Becke, J. Pulinthanath and EP. Rathgeb "On the use of concurrent multipath transfer over asymmetric paths," 3rd IEEE Global Telecommunications Conference (GLOBECOM 2010), pp. 1-6, 2010.

[6] T. Dreibholz, EP. Rathgeb, I. Rungeler, R. Seggelmann,M Tuxen and R. Stewartm "Stream Control Transmission Protocol: Past, Current and Future Standardization Activities," IEEE Communication Magazine, vol. 49, pp. 82-88, 2011.

[7] P. Natarajan, N. Ekiz, P. Amer and R. Stewart "Concurrent multipath transfer during path failure," Computer communication, vol. 32, pp. 1577-1587, 2009.

[8] E. Yilmaz, N. Ekiz, P. Natarajan, P. Amer, J. Leighton, F. Baker and R. Stewart "Throughput analysis of nonrenegable selective acknowledgments (NR-SACKs) for SCTP," Computer Communication, vol. 33, pp. 19821991, 2010.

[9] S. Shailendra, R. Bhattacharjee and SK. Bose "MPSCTP: A Simple and Efficient Multipath Algorithm for SCTP,"
IEEE Communication Letter, vol. 15, pp.1139-1141, 2011

[10] S. Shailendra, R. Bhattacharjee, SK. Bose "An implementation of Min-Max optimization for multipath SCTP through bandwidth estimation based resource pooling technique," International Journal of Electronics and Communication, vol. 67, pp. 246-249, 2013.

[11] S. Shailendra, R. Bhattacharjee and SK. Bose "A multipath variant of SCTP with optimized flow division extension," Computer Communication, vol. 67, pp. 56-65, 2015.

[12] C. Xu, T. Liu, J. Guan, H. Zhang and G. Muntean "CMTQA: Quality-Aware Adaptive Concurrent Multipath Data Transfer in Heterogeneous Wireless Networks," IEEE Transaction on Mobile Computing, vol. 12, pp. 21932205, 2013.

[13] A. Ford, C. Raiciu, M. Handley, S. Barre and J. Iyengar "Architectural guidelines for multipath TCP development," RFC6182, The Internet Engineering Task Force, 2011. https://tools.ietf.org/html/rfc6182

[14] C. Paasch, R. Khalili and O. Bonaventure "On the benefits of applying experimental design to improve multipath TCP," In Proceedings of the Ninth ACM Conference on Emerging Networking Experiments and Technologies, pp. 393-398, 2013.

[15] S. Barre, C. Paasch, and O. Bonaventure "Multipath TCP: from theory to practice," Networking 2011, pp. 444-457, 2011.

[16] M. Li, A. Lukyanenko, S. Tarkoma, Y. Cu and A. YlaPaaski "Tolerating path heterogeneity in multipath TCP with bounded receive buffers," In proceedings of the ACM SIGMETRICS/international conference on Measurement and modeling of computer systems, pp. 375-376, 2014.

[17] D. Zhou, W. Song and M. Shi "Goodput improvement for multipath TCP by congestion window adaptation in multiradio devices," In. Consumer Communications and Networking Conference (CCNC), pp. 508-514, 2013.

[18] N. Kuhn, E. Lochin, A. Mifdaoui, G. Sarwar, O. Mehani and R. Boreli "DAPS: Intelligent delay-aware packet scheduling for multipath transport," In: International Conference on Communications (ICC), pp. 1222-1227, 2014.

[19] Home Page of Network Simulator-2 (NS-2), http://www.isi.edu/nsnam/ns, [Accessed 15 May 2017]

[20] M. Nirmala and R. V. Pujeri "Performance of TCP Vegas, Bic and Reno Congestion Control Algorithms on Iridium Satellite Constellations" International Journal of Intelligent Systems and Applications (IJISA), vol. 12, pp 40-49, 2012.

[21] S. Sharma, S. Kumar, and B. Singh,"Hybrid Intelligent Routing in Wireless Mesh Networks: Soft Computing Based Approaches", International Journal of Intelligent Systems and Applications (IJISA), vol.6, no.1, pp.45-57, 2014.

[22] V. K. Sharma, S. S. P. Shukla, and V. Singh, "A tailored Q-Learning for routing in wireless sensor networks," In: 2nd IEEE International Conference on Parallel, Distributed and Grid Computing, pp. 663-668, Dec. 2012.

[23] V. K. Sharma, and M. Kumar, "Adaptive congestion control scheme in mobile ad-hoc networks," Peer-to-Peer Networking and Applications, vol. 10, no. 3, pp. 633-657, May 2017.

[24] L. P. Verma, and M. Kumar, "An adaptive data chunk scheduling for concurrent multipath transfer," Computer Standards \& Interfaces, vol. 52, pp. 97-104, May 2017.

[25] H.-Y. Hsie and R. Sivakumar, "pTCP: an end-to-end transport layer protocol for striped connections," In: Proceedings of the $10^{\text {th }}$ IEEE Conference on Network 
Protocols (ICNP), pp. 24-33, 2002.

[26] C.M. Huang and C.H. Tsai, "WiMP-SCTP: Multi-path transmission using stream control transmission protocol (SCTP) in wireless networks," In: Proceedings of the $21^{s t}$ IEEE Conference on Advanced Information Networking and Applications Workshops (AINAW), pp. 209-214, 2007.

[27] C. Raiciu, S. Barre, C. Pluntke, A. Greenhalgh, D. Wischik, and M. Handley, "Improving datacenter performance and robustness with multipath TCP", ACM SIGCOMM Computer Communication Review, vol. 41, no. 4, pp. 266-277, 2011.

[28] C. Raiciu, M. Handly and D. Wischik, "Coupled congestion control for multipath transport protocols", RFC 6356, Internet Engineering Task Force, 2011. https://tools.ietf.org/html/rfc6356 .

[29] M. A. Mansor, M. S. M. Kasihmuddin and S. Sathasivam, "Enhanced Hopfield Network for Pattern Satisfiability Optimization", International Journal of Intelligent Systems and Applications (IJISA), vol.8, no.11, pp.27-33, 2016.

[30] R. Janapati, C. Balaswamy, K. Soundararajan, "Enhancement of Indoor Localization in WSN using PSO tuned EKF", International Journal of Intelligent Systems and Applications (IJISA), vol.9, no.2, pp.10-17, 2017.

[31] V. C. Thang and N. V. Tao, "A Performance Evaluation of Improved IPv6 Routing Protocol for Wireless Sensor Networks", International Journal of Intelligent Systems and Applications (IJISA), vol.8, no.12, pp.18-25, 2016

\section{Authors' Profiles}

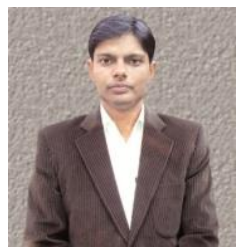

Lal Pratap Verma received his B.Tech. degree in Computer Science and Engineering from Dr. R.M.L. Avadh University, Faizabad, India in 2007 and M.Tech. degree in Information Technology from YMCA University of Science and Technology, Faridabad, India in 2011. He is currently working towards his Ph.D. degree in Computer Science and Engineering at Jaypee University of Engineering and Technology, Guna, India. His research interests include network systems, network security and Mobile Ad-hoc Network.

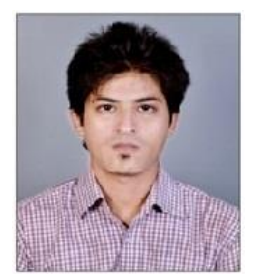

Varun Kumar Sharma received the Masters of Technology in Computer Science and Engineering from Jaypee University of Engineering and Technology, Guna, India and Bachelors of Engineering (Honors) in Computer Science and Engineering from Rajiv Gandhi Technical University, Bhopal, India. He is currently pursuing $\mathrm{Ph} . \mathrm{D}$. in Computer Science and Engineering from Jaypee University of Engineering and Technology, Guna, India. His research interests include performance evaluation of wireless network routing, cross-layer optimizations for ad-hoc and sensor networks, and energy aware routing.

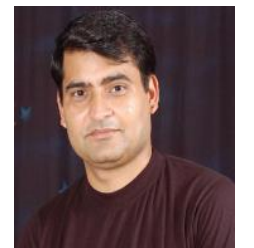

Mahesh Kumar is working as Assistant Professor in the Department of Computer Science and Engineering at Jaypee University of Engineering and Technology, Guna, Madhya Pradesh, India. He earned his $\mathrm{Ph}$. D. in Computer Science and Engineering, and M.Tech. in Information Technology. He has more than 11 years of experience in teaching and research. His current research interests include Wireless and Wired Networks, Internet Routing, Network Security, and IPv6.

How to cite this paper: Lal Pratap Verma, Varun Kumar Sharma, Mahesh Kumar "New Delay-based Fast Retransmission Policy for CMT-SCTP", International Journal of Intelligent Systems and Applications(IJISA), Vol.10, No.3, pp.59-66, 2018. DOI: 10.5815/ijisa.2018.03.07 